\title{
Nanosized sustained-release pyridostigmine bromide microcapsules: process optimization and evaluation of characteristics
}

This article was published in the following Dove Press journal:

International Journal of Nanomedicine

19 February 2013

Number of times this article has been viewed

\section{Qunyou Tan ${ }^{1, *}$ \\ Rong Jiang ${ }^{3, *}$ \\ Meiling $X u^{2,4, *}$ \\ Guodong Liu ${ }^{5, *}$ \\ Songlin $\mathrm{Li}^{\prime}$ \\ Jingqing Zhang ${ }^{2}$}

'Department of Thoracic Surgery, Institute of Surgery Research, Daping Hospital, Third Military Medical University, ${ }^{2}$ Medicine Engineering Research Center, Chongqing Medical University, ${ }^{3}$ Stem Cells and Tissue Engineering Research, Chongqing Medical University, ${ }^{4}$ Department of Pharmacy, Chongqing Emergency Medical Center, ${ }^{5}$ Eighth Department, Institute of Surgery Research, Daping Hospital, Third Military Medical University, Chongqing, People's Republic of China

*These authors contributed equally to this work
Correspondence: Jingqing Zhang Medicine Engineering Research Center, Chongqing Medical University, 50 I Building 22, Jingdi Garden, 6 Dahuang Road, Yuzhong District, Chongqing 400042, People's Republic of China Tel +86 I3308300303

Fax +86236876 7988

Email zjqrae0I@I63.com
Background: Pyridostigmine bromide (3-[[(dimethylamino)-carbonyl]oxy]-1-methylpyridinium bromide), a reversible inhibitor of cholinesterase, is given orally in tablet form, and a treatment schedule of multiple daily doses is recommended for adult patients. Nanotechnology was used in this study to develop an alternative sustained-release delivery system for pyridostigmine, a synthetic drug with high solubility and poor oral bioavailability, hence a Class III drug according to the Biopharmaceutics Classification System. Novel nanosized pyridostigmine-poly(lactic acid) microcapsules (PPNMCs) were expected to have a longer duration of action than free pyridostigmine and previously reported sustained-release formulations of pyridostigmine.

Methods: The PPNMCs were prepared using a double emulsion-solvent evaporation method to achieve sustained-release characteristics for pyridostigmine. The preparation process for the PPNMCs was optimized by single-factor experiments. The size distribution, zeta potential, and sustained-release behavior were evaluated in different types of release medium.

Results: The optimal volume ratio of inner phase to external phase, poly(lactic acid) concentration, polyvinyl alcohol concentration, and amount of pyridostigmine were $1: 10,6 \%$, $3 \%$ and $40 \mathrm{mg}$, respectively. The negatively charged PPNMCs had an average particle size of $937.9 \mathrm{~nm}$. Compared with free pyridostigmine, PPNMCs showed an initial burst release and a subsequent very slow release in vitro. The release profiles for the PPNMCs in four different types of dissolution medium were fitted to the Ritger-Peppas and Weibull models. The similarity between pairs of dissolution profiles for the PPNMCs in different types of medium was statistically significant, and the difference between the release curves for PPNMCs and free pyridostigmine was also statistically significant.

Conclusion: PPNMCs prepared by the optimized protocol described here were in the nanometer range and had good uniformity, with significantly slower pyridostigmine release than from free pyridostigmine. This novel sustained-release delivery nanosystem for pyridostigmine might alleviate the need to identify new acetylcholinesterase inhibitors.

Keywords: nanosized microcapsules, process optimization, characteristics, sustained-release, pyridostigmine bromide

\section{Introduction}

Pyridostigmine bromide (3-[[(dimethylamino)-carbonyl $]$ oxy $]$-1-methylpyridinium bromide), a reversible inhibitor of cholinesterase, has been used to treat myasthenia gravis, reverse neuromuscular blockade, and prevent nerve gas (ie, soman) poisoning. Pyridostigmine may also be used to treat flatulence and urinary retention after abdominal surgery. ${ }^{1-3}$

Pyridostigmine is readily soluble in water, which may be responsible for its short elimination half-life ( $1-2$ hours) and poor bioavailability $(11.5 \%-18.9 \%)^{4,5}$ Pyridostigmine tablets are given orally, and a treatment schedule of multiple doses 
every day (3-6 times per day) is recommended for adult patients, while sustained-release pyridostigmine $\left(\right.$ Timespan $\left.^{\circledR}\right)$ tablets can be taken once or twice daily. The results of an open-label multicenter clinical study support the usefulness of a sustained-release preparation of pyridostigmine to improve quality of life for patients with myasthenia gravis. ${ }^{6}$ The in vitro release characteristics of other modifiedrelease formulations of pyridostigmine reported previously (microparticles, pellets, and hydroxypropylmethylcellulosebased sustained-release tablet) are similar to those of Timespan. $^{7,8}$

Other approaches, including nanosized poly(lactic acid) (PLA) particles, are receiving attention ${ }^{9,10}$ in an effort to improve such sustained-release drug formulations further. In the present study, nanotechnology was used to develop an alternative sustained-release nanosystem for delivery of pyridostigmine, a synthetic drug with high solubility and poor oral bioavailability. Administered by the gastrointestinal or parenteral routes, this nanosystem could be expected to have a markedly longer duration of action compared with free pyridostigmine or previously reported sustainedrelease formulations of this drug. We prepared nanosized PLA microcapsules containing pyridostigmine (PPNMCs) using a double emulsion-solvent evaporation method, and single-factor experiments were done to formulate these microcapsules. ${ }^{11,12}$ The morphology of the PPNMCs was observed using biomicroscopy, particle size and zeta potential were characterized by dynamic light scattering, and in vitro release of pyridostigmine from PPNMCs was investigated using a dialysis method in different types of release medium in order to evaluate the sustained-release characteristics of PPNMCs in comparison with those of free pyridostigmine.

\section{Materials and methods}

\section{Materials}

Pyridostigmine bromide (purity 99.6\%) was purchased from Yuancheng Technology Development Co, Ltd (Wuhan, People's Republic of China), PLA (molecular weight 45,840-76,380) was supplied by the Research Center of Biomimetic Material Science and Engineering, Chongqing University, Chongqing, People's Republic of China, and polyvinyl alcohol (PVA)-217 was sourced from Kuraray Co, Ltd, Tokyo, Japan. All other chemical reagents were of analytical grade or better.

\section{Preparation of PPNMCs}

PPNMCs were produced using a modified $\mathrm{W}_{1} / \mathrm{O} / \mathrm{W}_{2}$ double emulsion-solvent evaporation method. ${ }^{13-16}$ Briefly, one milliliter of drug solution (containing $40 \mathrm{mg}$ of pyridostigmine) was added slowly to $10 \mathrm{~mL}$ of dichloromethane containing $6 \%$ w/v PLA under magnetic stirring (at about $3000 \mathrm{rpm}$ ) to yield a primary $\mathrm{W}_{1} / \mathrm{O}$ emulsion. The resulting emulsion was then dispersed in $100 \mathrm{~mL}$ of a $3 \% \mathrm{w} / \mathrm{v}$ PVA water solution $\left(\mathrm{W}_{2}\right)$ in an ice-water bath. The reaction proceeded under magnetic stirring for 30 minutes to form a homogeneous milky suspension $\left(\mathrm{W}_{1} / \mathrm{O} / \mathrm{W}_{2}\right.$ double emulsion), and the organic solvent was then evaporated off using a hypobaric drying method.

\section{Determination of percentage encapsulation and drug loading}

The nonentrapped pyridostigmine was separated from the PPNMCs by centrifugation at $12,000 \mathrm{~g}$ for 10 minutes. ${ }^{17}$ The supernatant was analyzed on a spectrophotometer (UV-5130, Shimadzu, Kyoto, Japan) and the empty PNMCs were used as the blank control (Figure 1). The absorption spectra of the PPNMCs and blank control clearly displayed considerable overlap, so direct ultraviolet-visible spectroscopy appeared unsuitable for determination of the pyridostigmine concentration. ${ }^{18,19}$ Fortunately, a second derivative spectroscopic method was able to be used for quantification (Figure 1). The ultraviolet-visible measurement conditions included a slit width of $8 \mathrm{~nm}$, a fast scan speed, and the recorder scale was expanded to facilitate reading a recording profile.

The amplitude of pyridostigmine was measured from baseline to the positive peak at $287 \mathrm{~nm}$, and the data were fitted to a linear regression equation:

$$
\mathrm{D}=0.0094 \mathrm{C}+0.00636
$$

where $r=0.9998(n=3), D$ is the pyridostigmine amplitude, and $\mathrm{C}$ was the concentration of pyridostigmine which ranged from $16.68 \mathrm{mg} / \mathrm{mL}$ to $38.92 \mathrm{mg} / \mathrm{mL}$.

Recovery of pyridostigmine was $100.30 \% \pm 0.94 \%$ $(n=9)$. Therefore, the second derivative spectroscopic method was shown to be an accurate, simple, and reliable analytical method. The following equations were used to calculate the entrapment efficiency (EE\%) and drug loading (DL\%). ${ }^{20}$

$$
\begin{gathered}
\text { EE }(\%)=\frac{\mathrm{W}_{\text {total drug added }}-\mathrm{W}_{\text {free drug }}}{\mathrm{W}_{\text {total drug added }}} \times 100 \% \\
\mathrm{DL}(\%)=\frac{\mathrm{W}_{\text {total drug added }}-\mathrm{W}_{\text {free drug }}}{\mathrm{W}_{\text {total drug added }}+\mathrm{W}_{\text {total PLA added }}} \times 100 \%
\end{gathered}
$$

where $\mathrm{W}_{\text {total drug added }}$ is the total amount of pyridostigmine added to the preparation, including both entrapped and free 
A

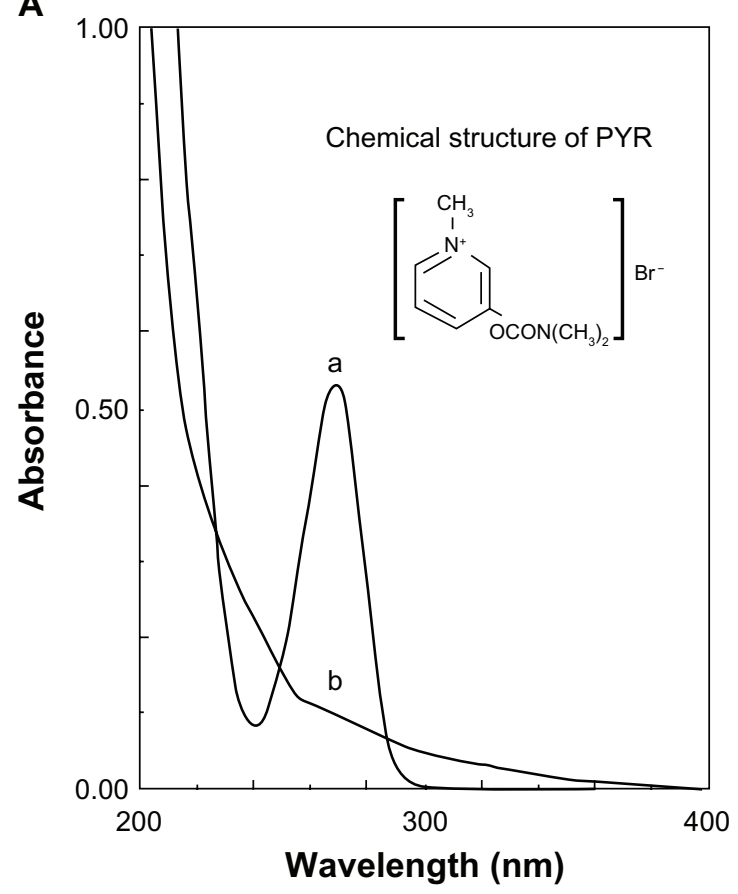

B

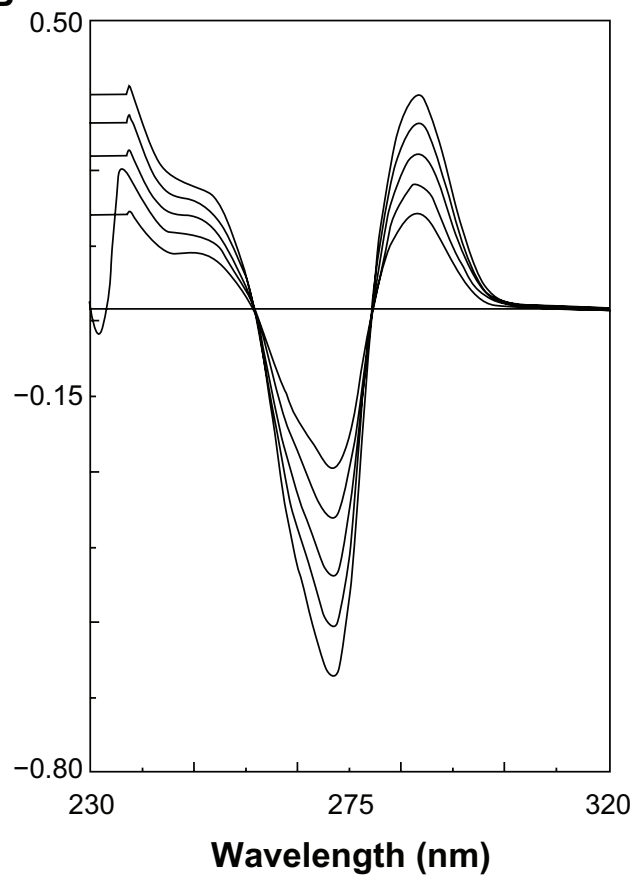

Figure I (A) Zero order spectra of the supernatant of nanosized pyridostigmine-poly(lactic acid) microcapsules (a) and empty nanosized microcapsules (b). (B) Second order derivative spectra of pyridostigmine.

Abbreviation: PYR, pyridostigmine.

pyridostigmine, and $\mathrm{W}_{\text {free drug }}$ is the amount of free pyridostigmine, and $\mathrm{W}_{\text {total PLA added }}$ is the total amount of PLA added to the preparation.

\section{Optimization of formulation component}

The previous literature and our preliminary studies showed that some formulation factors have key roles in the encapsulation rate and drug loading capacity of nanosized PLA delivery systems. $^{21,22}$ In our study, single-factor tests were performed to identify the critical parameters of the formulation and the preparation process used for PPNMCs. In other words, one parameter was subjected to change while keeping the others constant. First, the type of organic solvent (external phase of the primary emulsion) was optimized, while keeping the volume ratio of internal phase to external phase (1:10), PLA concentration $(2 \%, \mathrm{w}: \mathrm{v})$, PVA concentration $(1 \%, \mathrm{w}: \mathrm{v})$ and amount of pyridostigmine $(10 \mathrm{mg})$ constant. Second, the volume ratio of inner water phase to external phase of primary emulsion was changed in order to select the optimum level while using dichloromethane as oil phase, and keeping the PLA concentration (2\%, w:v), PVA concentration (1\%,w:v) and amount of pyridostigmine $(10 \mathrm{mg})$ constant. Third, the PLA concentration was optimized while using dichloromethane as oil phase, and keeping the volume ratio of internal phase to external phase (1:10), PVA concentration $(1 \%, \mathrm{w}: \mathrm{v})$ and amount of pyridostigmine $(10 \mathrm{mg})$ constant. Fourth, the PVA concentration was optimized while using dichloromethane as oil phase, and keeping the volume ratio of internal phase and external phase (1:10), PLA concentration (6\%,w:v) and amount of pyridostigmine $(10 \mathrm{mg})$ constant. Finally, the amount of pyridostigmine was optimized while using dichloromethane as oil phase, and keeping the volume ratio of internal phase to external phase (1:10), PLA concentration $(6 \%, \mathrm{w}: \mathrm{v})$, and PVA concentration $(3 \%, \mathrm{w}: \mathrm{v})$ constant.

In this way, several different concentrations of critical preparation factors (such as oil phase type and the volume ratio of internal phase to external phase of the primary emulsion) and the main formulation components (ie, PLA, PVA, and pyridostigmine concentrations) were tested one by one to optimize the properties of the formulation, and PPNMCs with various formulations were prepared according to a one-factor experimental design. PPNMCs having good structural integrity and showing maximum entrapment and suitable drug loading were then studied further.

\section{Morphological features}

A sample was prepared by diluting $1 \mathrm{~mL}$ of the PPNMCs with $9 \mathrm{~mL}$ of distilled water. Its appearance was observed by optical biomicroscopy (XSP-35-1600X, Phoenix, Shangrao, People's Republic of China) and micrographs were taken using a camera (C-60 ZOOM, Olympus, Hong Kong, People's Republic of China). 


\section{Particle size and electric charge}

The samples were prepared using the same method as described above. PPNMC particle sizes and their distribution were determined by dynamic light scattering (Zeta-Sizer Nano-ZS90, Malvern, Worcestershire, UK). The zeta potentials and their width were measured using the same equipment. ${ }^{16}$

\section{In vitro release of pyridostigmine from PPNMCs}

The in vitro release studies were performed using a modified dialysis method. ${ }^{11}$ PPNMCs equivalent to $10 \mathrm{mg}$ of pyridostigmine were placed in dialysis tubes and then immersed into the diffusion medium. The devices were run at $100 \mathrm{rpm}$ and maintained at $37^{\circ} \mathrm{C} \pm 0.5^{\circ} \mathrm{C}$. At designated time points, a $0.5 \mathrm{~mL}$ quantity of diffusion medium was withdrawn, followed by supplementing with an equal volume of fresh diffusion medium to keep the total volume of diffusion medium constant. Quantitative determination of pyridostigmine was carried out using second derivative spectrophotometry. The in vitro release experiments were conducted in three batches each of PPNMCs and pure pyridostigmine. The in vitro release kinetics of pyridostigmine from the nanosized microcapsules were investigated in four different types of dissolution medium: $\mathrm{pH} 7.4$ phosphate buffer solution ( $\mathrm{pH}$ similar to that of blood); $0.1 \mathrm{~mol} / \mathrm{L} \mathrm{HCl}$ ( $\mathrm{pH} 1.2$, similar to that of gastric fluid); $\mathrm{pH} 6.8$ phosphate buffer solution ( $\mathrm{pH}$ similar to that of intestinal fluid); and $0.1 \mathrm{~mol} / \mathrm{L} \mathrm{HCl}$ for the first 2 hours and $\mathrm{pH} 6.8$ phosphate buffer for the next 70 hours.

\section{Results and discussion Preparation of PPNMCs}

The various types of solvents, namely dichloromethane, ethyl acetate, mixed solvent (dichloromethane to ethyl acetate, $1: 1, \mathrm{v}: \mathrm{v})$, and acetone, were screened for suitability in the preparation of PPNMCs. No PPNMC was formed when acetone was used as oil phase. Further, only a few PPNMCs formed when ethyl acetate and the mixed solvent were used. Fortunately, many PPNMCs formed when dichloromethane was used as oil phase, so dichloromethane was chosen to prepare the PPNMCs in our study (Figure 2A).

As shown in Figure 2B, the optimum volume ratio of internal phase to external phase was calculated to be 1:10, because it gave a high encapsulation rate of $30.44 \%$ (mean, $\mathrm{n}=3$ ) and a high drug-loading capacity of $1.45 \%$. The main constituents of PPNMCs, namely PLA as an encapsulation material and PVA as a stabilizing agent and surfactant, were chosen at certain concentrations to achieve desirable formulation properties. As seen in Figure 2C, on increasing the PLA concentration, entrapment efficiency increased up to $44.80 \%$ and drug-loading capacity decreased to $0.64 \%$. Drug-loading capacity was maintained at around $0.70 \%$ when the PLA concentration was varied from $2 \%$ to $6 \%$. As seen in Figure 2D, a PVA concentration of 5\% achieved the maximum entrapment efficiency (83.16\%) and drug-loading capacity (3.92\%), but obvious aggregation was observed under this condition. Therefore, a PVA concentration of 3\% was chosen to obtain PPNMCs with relatively high entrapment and drug loading as well as good surface morphology. When the amount of pyridostigmine was set at $40 \mathrm{mg}$, the maximum entrapment efficiency and drug-loading capacity achieved was $66.35 \%$ and $4.21 \%$, respectively (Figure 2E). Therefore, PPNMCs might have limited payload and encapsulation capacity when the formulation components are kept constant.

Taken together, the above findings suggest that the optimal oil phase type, volume ratio of inner phase to external phase, PLA concentration (\%, w:v), PVA concentration (\%,w:v) and amount of pyridostigmine should be dichloromethane, $1: 10,6 \%, 3 \%$, and $40 \mathrm{mg}$, respectively.

\section{Validation of model optimization}

In order to evaluate the entrapment and drug loading of the optimized formulations obtained by the single-factor tests, PPNMCs were prepared using the protocol described earlier (with dichloromethane used as oil phase, volume ratio of inner phase to external phase, PLA concentration, PVA concentration, and amount of pyridostigmine set at $1: 10,6 \%, 3 \%$, and $40 \mathrm{mg}$, respectively). Encapsulation rates and drug loading of pyridostigmine in PPNMCs prepared under these optimized conditions were found to be $67.59 \% \pm 1.46 \%$ and $4.31 \% \pm 0.17 \%$, respectively $(\mathrm{n}=3)$.

The encapsulation rates of the optimized PPNMCs were higher than those of nanosized PLA particles reported previously to range from $2.6 \%$ to $65.3 \% .^{16,23-27}$ In addition, the drug-loading capacities of the optimized PPNMCs prepared here were mid value compared with that of other nanosized PLA particles reported earlier to range from $0.14 \%$ to $8 \% .{ }^{23,25-}$ ${ }^{27}$ These changes in entrapment efficiency and drug-loading capacity might be due to the different type, amount, and solubility properties of the agents used, as well as the different preparation methods used and aims of the experiments. In our study, PPNMCs were planned and produced, and enabled good sustained release of pyridostigmine. Our hypothesis was confirmed by these in vitro experiments. 


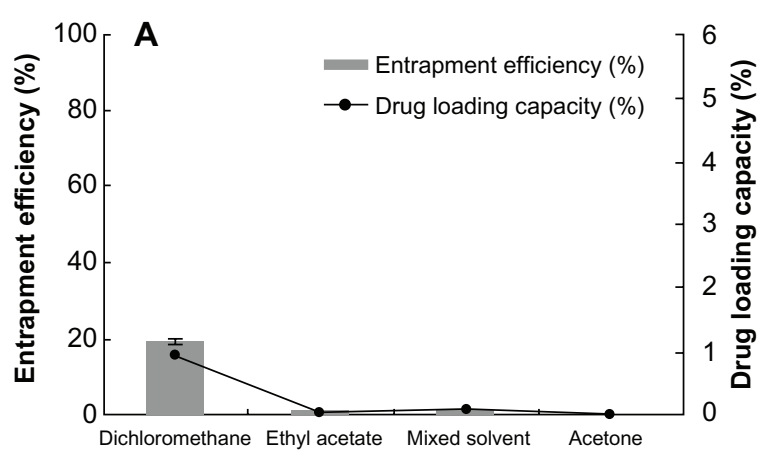

Solvent type

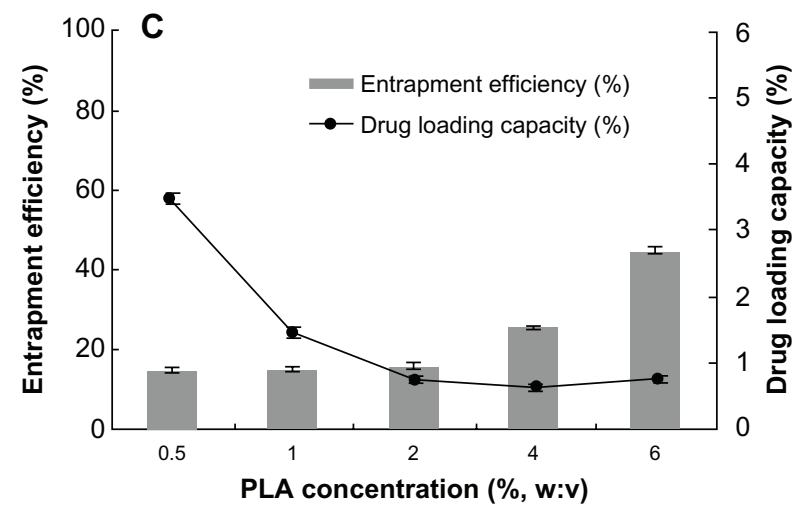

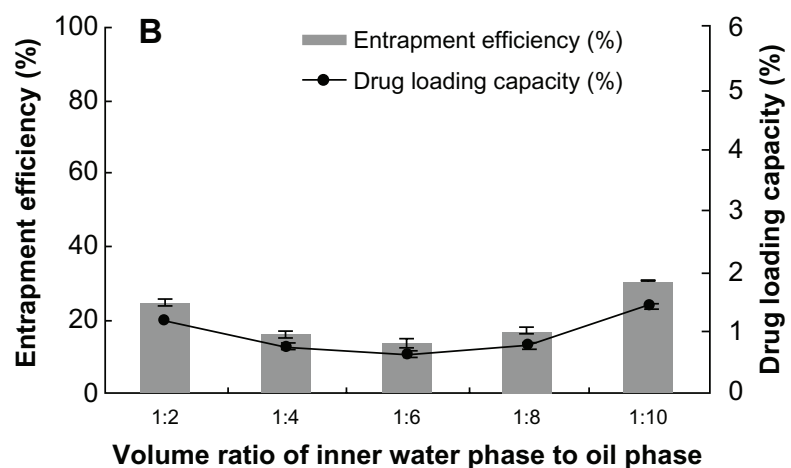

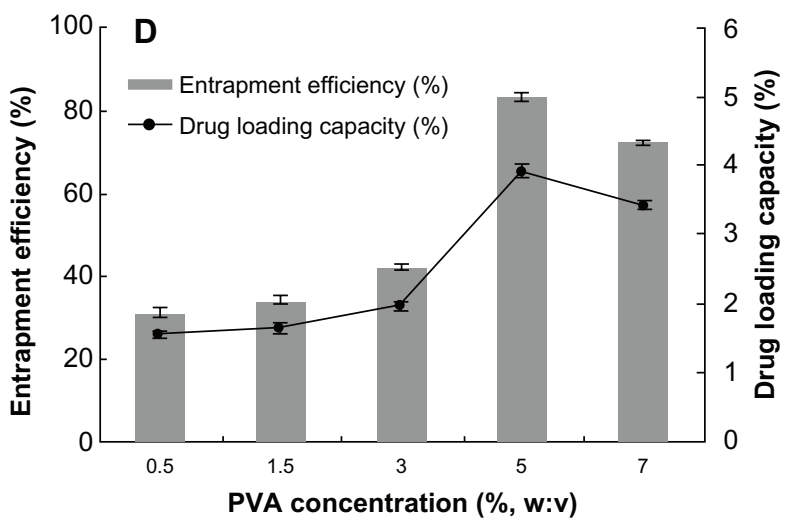

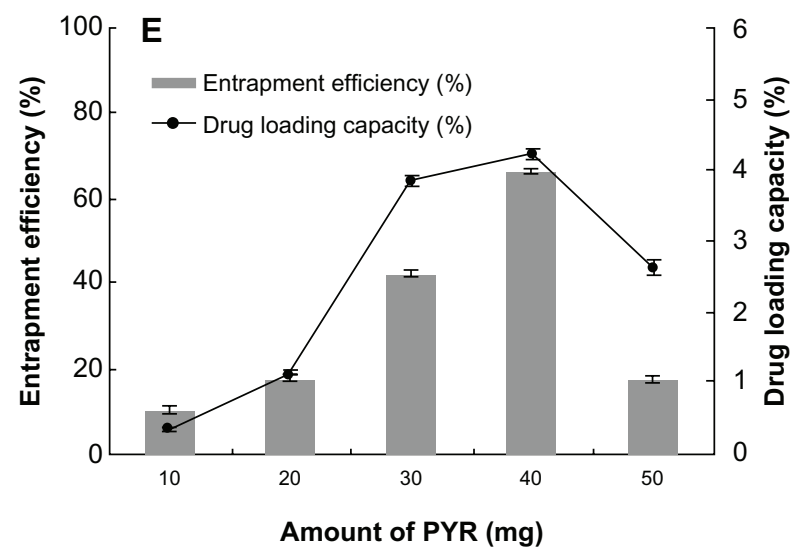

Figure 2 Effect of (A) solvent type, (B) volume ratio of inner water phase to oil phase, (C) PLA concentration, (D) PVA concentration, and (E) amount of PYR on entrapment efficiency and drug-loading capacity $(n=3)$.

Abbreviations: PYR, pyridostigmine; PLA, poly(lactic acid); PVA, polyvinyl alcohol.

\section{Characteristics of PPNMCs}

As shown in Figure 3A, PPNMCs were evenly dispersed in distilled water, and had a round appearance. The structure of the PPNMCs remained relatively intact with no obvious aggregation. The mean particle diameter of the smooth spherical PPNMCs was $937.9 \pm 2.5 \mathrm{~nm}$ and the mean polydispersity index (indicating size distribution) was $0.722 \pm 0.02$ (Figure 3B). The nanoscale size range and good homogeneity of the PPNMCs might be attributable to the main constituents of the designed nanosized microcapsules. The encapsulation material (PLA) intercalated with the stabilizing agent and surfactant (PVA) also contributed to the production of the double emulsion with the desired interfacial strength and effective stress. The zeta potentials and width of the optimized formulations were $-19.55 \pm 2.1 \mathrm{mV}$ and $4.7 \pm 0.5 \mathrm{mV}$, respectively (Figure 3C). The electronegativity of the PPNMCs might be explained by the presence of terminal carboxylic groups in the polymers. The high electric potentials might ensure a high energy barrier to stabilize the nanosystem. In other words, charge repulsion provided an electrostatic potential barrier for the PPNMCs and thus good stability, making these nanoparticle systems a good choice for slow delivery of pyridostigmine. ${ }^{28}$ 


\section{In vitro release of pyridostigmine from PPNMCs}

Release curves were achieved by measuring cumulative drug release for up to 72 hours. Figure 4 shows the drug release curves for the nanosized PPNMCs in four types of dissolution medium with different $\mathrm{pH}$ levels: $\mathrm{pH} 7.4$ phosphate buffer solution, $0.1 \mathrm{~mol} / \mathrm{L} \mathrm{HCL}$, pH 6.8 phosphate buffer solution, and $0.1 \mathrm{~mol} / \mathrm{L} \mathrm{HCl}$ (2 hours) and $\mathrm{pH} 6.8$ phosphate buffer solution (70 hours). Several mathematical models fitted with these experimental data. As shown in Table 1, the results indicate that both the Ritger-Peppas and Weibull models fitted well, regardless of type of dissolution medium used..$^{29}$ The PPNMCs showed an initially rapid rate of release of pyridostigmine followed by slower release over an extended period of time ( 72 hours). About half of the total amount of pyridostigmine was released from the PPNMCs in the first 30 minutes, because of the mass of nonentrapped pyridostigmine. ${ }^{30,31}$ This "burst effect" enables rapid drug efficacy for patients in the clinical setting. On the other hand, the entrapped pyridostigmine was prevented from diffusing into the dissolution medium by the PLA nanoparticles, and this might be responsible for the slow release rate after 30 minutes, whereas for the free pyridostigmine solution, under the same conditions, $85.44 \% \pm 0.18 \%(n=3)$ was released in the first 30 minutes and $99.94 \% \pm 0.01 \%(n=3)$ over 24 hours. The rate of release of pyridostigmine from the PPNMCs was therefore relatively slow compared with that of free pyridostigmine.

Among several methods investigated for comparison of dissolution profiles, the similar factor $\left(f_{2}\right)$ method is the simplest and most reliable, so was used in our experiments. The $f_{2}$ values were calculated using the following formula:

$$
f_{2}=50 \lg \left\{\left[1+(1 / n) \sum_{i=1}^{n} W_{t}\left(\overline{X_{i i}}-\overrightarrow{X_{r i}}\right)^{2}\right]^{-1 / 2} \times 100\right\}
$$

where $f_{2}$ is the similar factor, $\overline{X_{i i}}$ and $\overrightarrow{X_{r i}}$ are the cumulative drug release rates at time $t$ of two dissolution curves, $n$ is the number of sample points, and $W_{t}$ is the weight, and set as 1 here.

When the two profiles were identical, the $f_{2}$ value was 100 . In the case of an average difference of $10 \%$ at all sampling time points, the $f_{2}$ value changed to 50 . The US Food and Drug Administration has set a common criterion for the $f_{2}$ value (50-100) to illustrate similarity between a pair of dissolution curves. The higher the value of a similar factor, the closer the similarity. As shown in Table 2, a statistically significant similarity was found between every pair of dissolution profiles for PPNMCs in the different types of dissolution medium. On the other hand, there was a significant difference between two curves when the $f_{2}$ value was below 50 . The difference between the curves for PPNMCs and pure pyridostigmine was highly statistically significant.

Overall, the above results confirm that the release rates for pyridostigmine, a highly water-soluble drug, can be controlled by entrapping the drug in nanosized PLA microcapsules. Our findings suggest that PPNMCs might be useful carriers for sustained-release pyridostigmine in the treatment of myasthenia gravis.

\section{Conclusion}

This study explored the feasibility of formulating a biodegradable nanosized microcapsule pyridostigmine delivery system using a double emulsion-solvent evaporation technique. Highly water-soluble pyridostigmine could
A

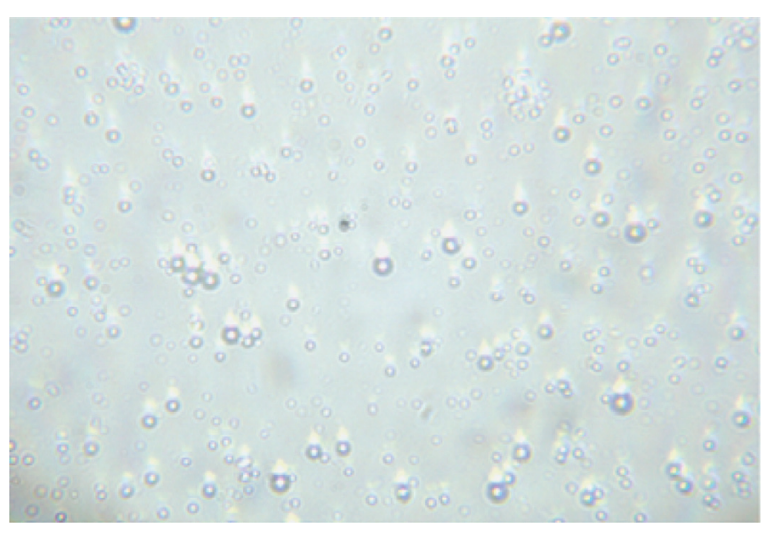

B

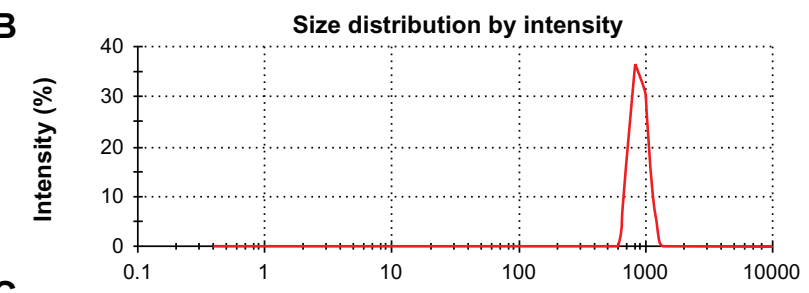

C

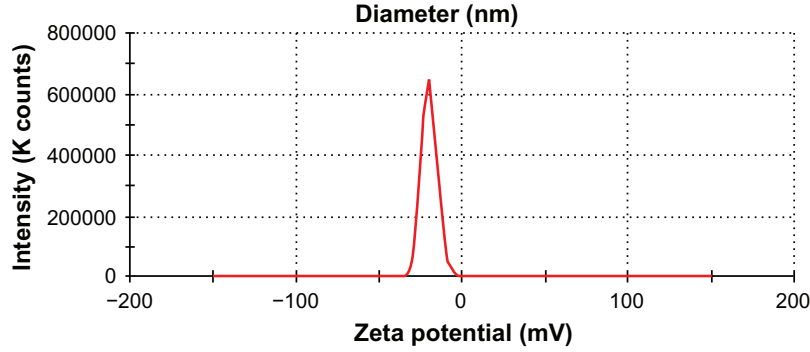

Figure 3 (A) Photomicrographs (2000x), (B) graph depicting particle size distribution, and (C) graph depicting zeta potential of nanosized pyridostigmine-poly(lactic acid) microcapsules. 


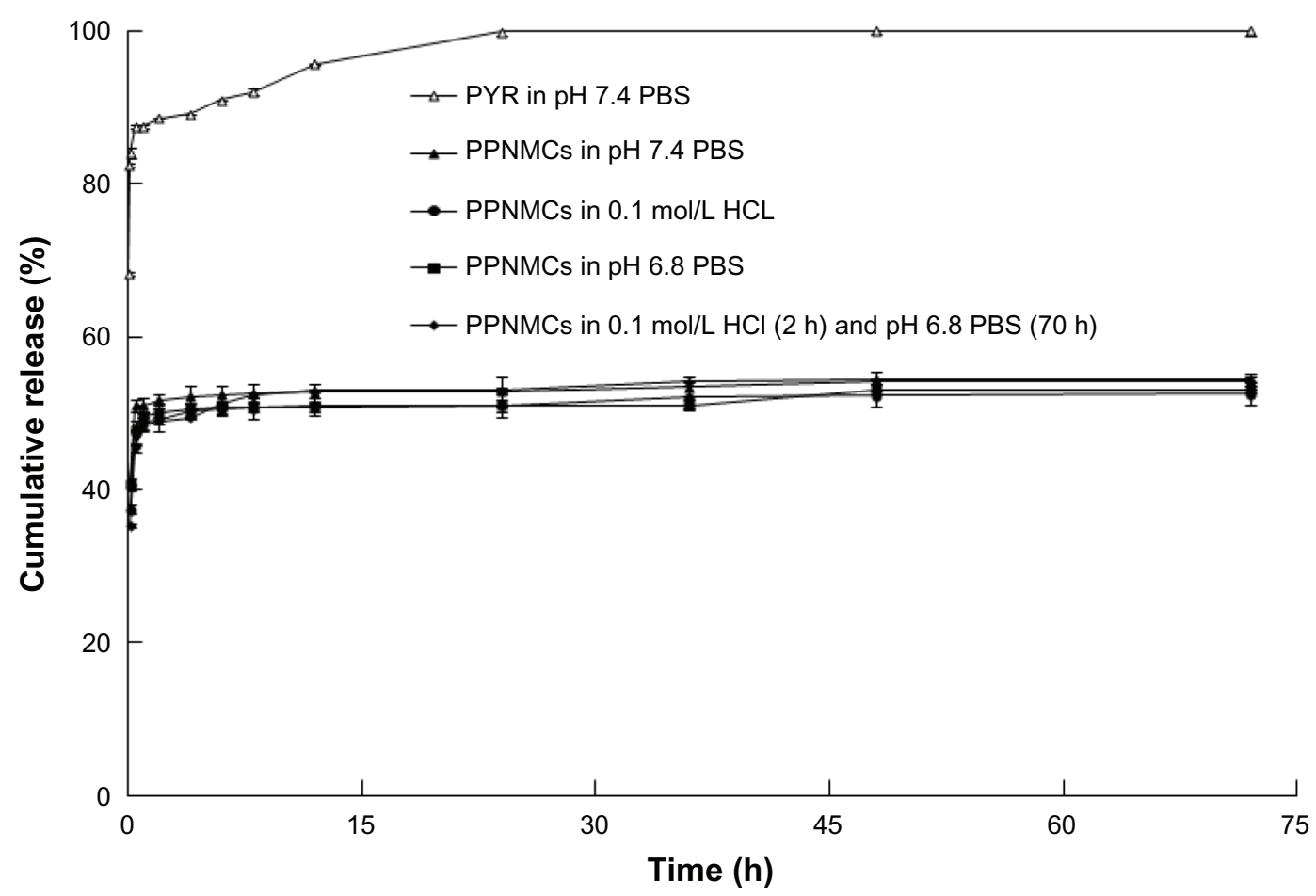

Figure 4 Drug release profiles of pure pyridostigmine in dissolution medium (pH 7.4 PBS), and PPNMCs in four types of dissolution medium (pH 7.4 PBS, 0.I mol/L HCL, $\mathrm{pH}$ 6.8 PBS, and $0.1 \mathrm{~mol} / \mathrm{L} \mathrm{HCl}$ for 2 hours and subsequently $\mathrm{pH} 6.8 \mathrm{PBS}$ for 70 hours).

Abbreviations: PPNMCs, nanosized pyridostigmine-poly(lactic acid) microcapsules; PBS, phosphate buffer solution; PYR, pyridostigmine.

be entrapped well in nanosized PLA microcapsules, the properties of which could be optimized by changing various formulation and process parameters.

In the present work, the process optimization of PPNMCs was carried out using a single-factor test methodology. Our study confirmed that the oil phase, volume ratio of inner phase to external phase, PLA concentration, PVA concentration, and pyridostigmine content has a significant impact on the entrapment and drug payload of PPNMCs. PPNMCs prepared using the optimized protocol were in the nanometer range, with

Table I Mathematical model of mean cumulative release rate versus time

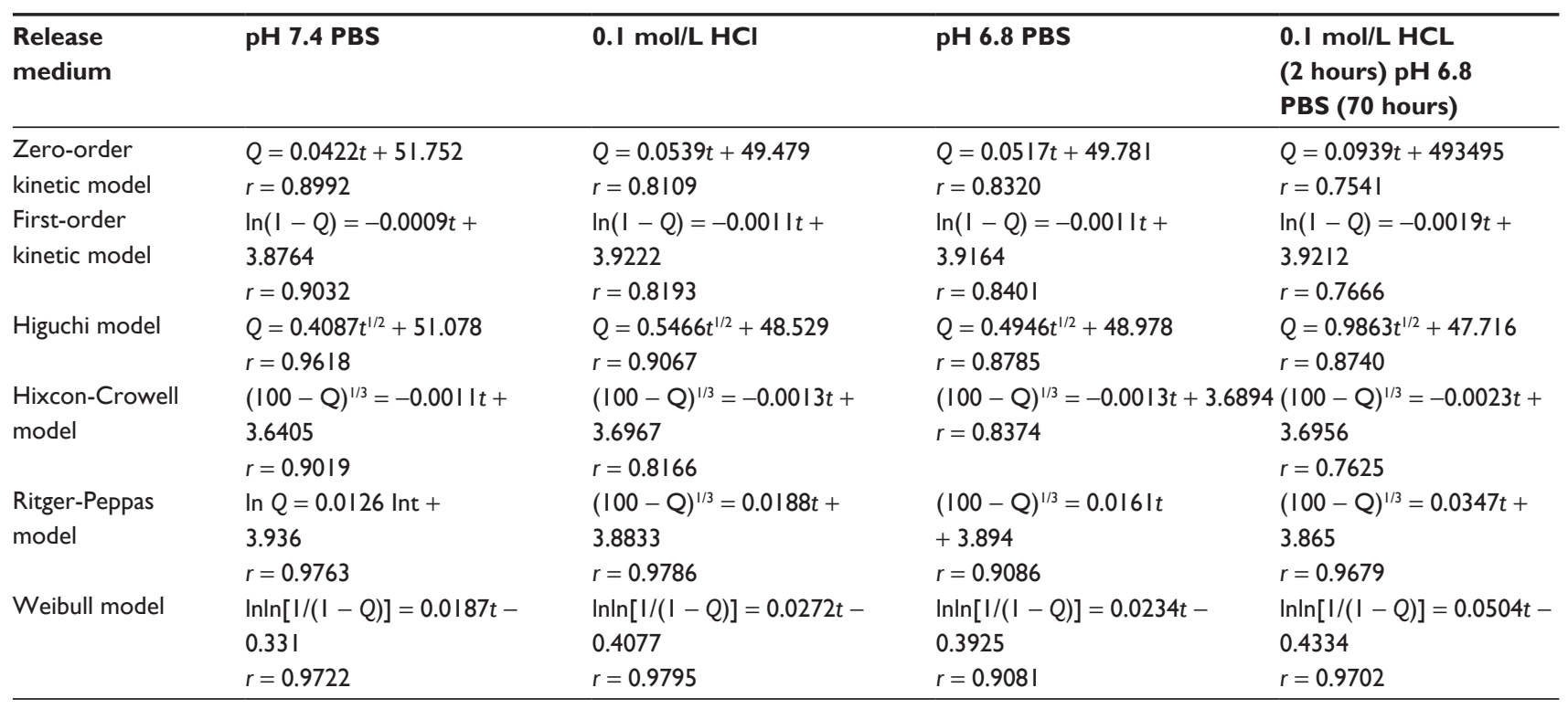

Note: $Q$ means cumulative pyridostigmine release at time $t$.

Abbreviation: PBS, phosphate-buffered solution. 
Table 2 Similarity between dissolution profiles of free pyridostigmine and PPNMCs in different types of dissolution medium

\begin{tabular}{|c|c|c|c|c|}
\hline \multicolumn{2}{|c|}{ Release profile I } & \multicolumn{2}{|c|}{ Release profile 2} & \multirow[t]{2}{*}{$f_{2}$} \\
\hline Formulation & Release medium & Formulation & Release medium & \\
\hline PPNMCs & $0.1 \mathrm{~mol} / \mathrm{L} \mathrm{HCL}$ & Free PYR & $\mathrm{pH} 7.4$ PBS & $18.4 \mid$ \\
\hline PPNMCs & $\mathrm{pH} 7.4$ PBS & Free PYR & $\mathrm{pH} 7.4$ PBS & 19.58 \\
\hline PPNMCs & $\mathrm{pH} 6.8 \mathrm{PBS}$ & Free PYR & $\mathrm{pH} 7.4$ PBS & 18.75 \\
\hline \multirow[t]{2}{*}{ PPNMCs } & $0.1 \mathrm{~mol} / \mathrm{L} \mathrm{HCL}$ (2 hours) & Free PYR & $\mathrm{pH} 7.4$ PBS & 18.56 \\
\hline & and $\mathrm{pH} 6.8$ PBS (70 hours) & & & \\
\hline PPNMCs & $0.1 \mathrm{~mol} / \mathrm{L} \mathrm{HCL}$ & PPNMCs & $\mathrm{pH} 7.4$ PBS & 79.65 \\
\hline PPNMCs & $0.1 \mathrm{~mol} / \mathrm{L} \mathrm{HCL}$ & PPNMCs & $\mathrm{pH} 6.8$ PBS & 90.94 \\
\hline \multirow[t]{2}{*}{ PPNMCs } & $0.1 \mathrm{~mol} / \mathrm{L} \mathrm{HCL}$ & PPNMCs & $0.1 \mathrm{~mol} / \mathrm{L} \mathrm{HCL}$ (2 hours) & 86.40 \\
\hline & & & and $\mathrm{pH} 6.8$ PBS (70 hours) & \\
\hline PPNMCs & $\mathrm{pH} 7.4$ PBS & PPNMCs & $\mathrm{pH} 6.8$ PBS & 84.64 \\
\hline \multirow[t]{2}{*}{ PPNMCs } & $\mathrm{pH} 7.4$ PBS & PPNMCs & $0.1 \mathrm{~mol} / \mathrm{L} \mathrm{HCL}$ (2 hours) & 75.59 \\
\hline & & & and pH 6.8 PBS (70 hours) & \\
\hline \multirow[t]{2}{*}{ PPNMCs } & $\mathrm{pH} 6.8$ PBS & PPNMCs & $0.1 \mathrm{~mol} / \mathrm{L} \mathrm{HCL}$ (2 hours) & 80.05 \\
\hline & & & and $\mathrm{pH} 6.8$ PBS (70 hours) & \\
\hline
\end{tabular}

Abbreviations: PBS, phosphate-buffered saline; PYR, pyridostigmine; PPNMCs, nanosized pyridostigmine-poly(lactic acid) microcapsules; $f_{2}$, similar factor.

good uniformity in size, spherical shape, and a smooth surface. The release tests were conducted in different types of release medium. Compared with free pyridostigmine, PPNMCs had significantly slower release rates of pyridostigmine in vitro. Further studies are necessary to evaluate the relationship between release behavior in vitro and in vivo. The preliminary results of our research on PPNMCs as a sustained delivery nanosystem are very encouraging. Development of this novel pyridostigmine sustained-release delivery nanosystem might relieve the immediate pressure to find new acetylcholinesterase inhibitors.

\section{Acknowledgments}

This research was partially supported by grants from the National Natural Science Foundation of China (30973645), Specialized Research Fund for the Doctoral Program of Higher Education (20095503120008), Chongqing Natural Science Foundation (CSTC2012 JJB10027), and Chongqing Education Committee Fund (Excellent University Personnel Financial Aid Plan, KJ120321).

\section{Disclosure}

The authors report no conflicts of interest in this work.

\section{References}

1. Andersen JB, Engeland A, Owe JF, Gilhus NE. Myasthenia gravis requiring pyridostigmine treatment in a national population cohort. Eur J Neurol. 2010;17:1445-1450.

2. Maselli RA, Henderson JD, Ng J, Follette D, Graves G, Wilson BW. Protection of human muscle acetylcholinesterase from soman by pyridostigmine bromide. Muscle Nerve. 2011;43:591-595.

3. Tan Q, Hu N, Liu G, et al. Role of a novel pyridostigmine bromide-phospholipid nanocomplex in improving oral bioavailability. Arch Pharm Res. 2012;35:499-508.
4. Breyer-Pfaff U, Maier U, Brinkmann AM, Schumm F. Pyridostigmine kinetics in healthy subjects and patients with myasthenia gravis. Clin Pharmacol Ther. 1985;37:495-501.

5. White MC, De Silva P, Havard CW. Plasma pyridostigmine levels in myasthenia gravis. Neurology. 1981;31:145-150.

6. Sieb JP, Köhler W. Benefits from sustained-release pyridostigmine bromide in myasthenia gravis: results of a prospective multicenter open-label trial. Clin Neurol Neurosurg. 2010;112:781-784.

7. Hegazy N, Demirel M, Yazan Y. Preparation and in vitro evaluation of pyridostigmine bromide microparticles. Int J Pharm. 2002;242: 171-174.

8. Huang YT, Tsai TR, Cheng CJ, Cham TM, Lai TF, Chuo WH. Formulation design of an HPMC-based sustained release tablet for pyridostigmine bromide as a highly hygroscopic model drug and its in vivo/in vitro dissolution properties. Drug Dev Ind Pharm. 2007;33:1183-1191.

9. Adair JH, Parette MP, Altinoğlu EI, Kester M. Nanoparticulate alternatives for drug delivery ACS Nano. 2010;4:4967-4970.

10. Fu J, Wang D, Wang T, et al. High entrapment efficiency of chitosan/ polylactic acid/tripolyphotspate nanosized microcapsules for rapamycin by an emulsion-evaporation approach. J Biomed Nanotechnol. 2010;6: 725-728.

11. Tan QY, Wang N, Yang H, et al. Preparation and characterization of lipid vesicles containing uricase. Drug Deliv. 2010;17:28-37.

12. Silva AT, Nguyen A, Ye C, Verchot J, Moon JH. Conjugated polymer nanoparticles for effective siRNA delivery to tobacco BY-2 protoplasts. BMC Plant Biol. 2010;10:291.

13. Freitas MN, Marchetti JM. Nimesulide PLA microspheres as a potential sustained release system for the treatment of inflammatory diseases. Int J Pharm. 2005;295:201-211.

14. Chaisria W, Hennink WE, Okonogi S. Preparation and characterization of cephalexin loaded PLGA microspheres. Curr Drug Deliv. 2009;6: 69-75.

15. Cui F, Cun D, Tao A, et al. Preparation and characterization of melittinloaded poly(DL-lactic acid) or poly (DL-lactic-co-glycolic acid) microspheres made by the double emulsion method. J Control Release. 2005;107:310-319.

16. Musumeci T, Ventura CA, Giannone L, et al. PLA/PLGA nanoparticles for sustained release of docetaxel. Int J Pharm. 2006;325:172-179.

17. Zhang JQ, Liu J, Li XL, Jasti BR. Preparation and characterization of solid lipid nanoparticles containing silibinin. Drug Deliv. 2007;14: 381-387.

18. Rodenas V, Parra A, Garcia-Villanova J, Gomez MD. Simultaneous determination of cefepime and L-arginine in injections by second-derivative spectrophotometry. J Pharm Biomed Anal. 1995;13:1095-1099. 
19. El-Gindy A, Emara S, Hadad GM. Determination of certain drugs in binary mixtures formulations by second derivative ratio spectrophotometry and LC. Farmaco. 2004;59:703-712.

20. Zhang JQ, Zhang ZR, Yang H, Tan QY, Qing SR, Qiu XL. Lyophilized paclitaxel magnetoliposomes as a potential drug delivery system for breast carcinoma via parenteral administration: in vitro and in vivo studies. Pharm Res. 2005;22:573-583.

21. Liu J, Qiu Z, Wang S, Zhou L, Zhang S. A modified double-emulsion method for the preparation of daunorubicin-loaded polymeric nanoparticle with enhanced in vitro anti-tumor activity. Biomed Mater. 2010;5:065002.

22. Nagarwal RC, Singh PN, Kant S, Maiti P, Pandit JK. Chitosan coated PLA nanoparticles for ophthalmic delivery: characterization, in-vitro and in-vivo study in rabbit eye. J Biomed Nanotechnol. 2010;6:648-657.

23. Kumari A, Yadav SK, Pakade YB, et al. Nanoencapsulation and characterization of Albizia chinensis isolated antioxidant quercitrin on PLA nanoparticles. Colloids Surf B Biointerfaces. 2011;82:224-232.

24. Lan $\mathrm{CH}$, Rémi $\mathrm{F}$, Yves W. Increase in stability and change in supramolecular structure of b-carotene through encapsulation into polylactic acid nanoparticles. Food Chem. 2011;124:42-49.

25. Kunii R, Onishi H, Machida Y. Preparation and antitumor characteristics of PLA/(PEG-PPG-PEG) nanoparticles loaded with camptothecin. Eur J Pharm Biopharm. 2007;67:9-17.
26. Hou ZQ, Zhou CX, Luo Y, et al. PLA nanoparticles loaded with an active lactone form of hydroxycamptothecin: development, optimization, and in vitro-in vivo evaluation in mice bearing h22 solid tumor. Drug Dev Res. 2011;72:1-9.

27. Leo E, Brina B, Forni F, Vandelli MA. In vitro evaluation of PLA nanoparticles containing a lipophilic drug in water-soluble or insoluble form. Int J Pharm. 2004;278:133-141.

28. Tan QY, Wang N, Yang H, et al. Characterization, stabilization and activity of uricase loaded in lipid vesicles. Int J Pharm. 2010;384: 165-172.

29. Tan Q, Li Y, Wu J, Mei H, Zhao C, Zhang J. An optimized molecular inclusion complex of diferuloylmethane: enhanced physical properties and biological activity. Int J Nanomedicine. 2012;7:5285-5393.

30. Magenheim B, Levy MY, Benita S. A new in vitro technique for evaluation of drug release profile from colloidal carriers-ultrafiltration technique at low pressure. Int J Pharm. 1993;94:115-123.

31. He D, Deng P, Yang L, et al. Molecular encapsulation of rifampicin as an inclusion complex of hydroxypropyl- $\beta$-cyclodextrin: Design; characterization and in vitro dissolution. Colloids Surf B Biointerfaces 2012;103C:580-585.
International Journal of Nanomedicine

\section{Publish your work in this journal}

The International Journal of Nanomedicine is an international, peerreviewed journal focusing on the application of nanotechnology in diagnostics, therapeutics, and drug delivery systems throughou the biomedical field. This journal is indexed on PubMed Central,

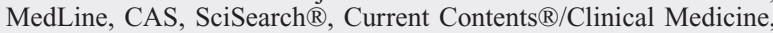

\section{Dovepress}

Journal Citation Reports/Science Edition, EMBase, Scopus and the Elsevier Bibliographic databases. The manuscript management system is completely online and includes a very quick and fair peer-review system, which is all easy to use. Visit http://www.dovepress.com/ testimonials.php to read real quotes from published authors. 